\title{
Predicting Blood Temperature using Gaussian Processes
}

\author{
Fabián Güiza $^{1}$, Jan Ramon ${ }^{1}$, Geert Meyfroidt ${ }^{2}$, Hendrik Blockeel ${ }^{1}$, Maurice \\ Bruynooghe ${ }^{1}$, and Greet Van Den Berghe ${ }^{2}$ \\ 1 Department of Computer Science, K.U.Leuven \\ 2 Department of intensive care medicine, University Hospital, Leuven
}

Background: One of the requirements for an ICU patient to be considered in an inflammatory state (such as SIRS) is that his temperature fall within a given range (typically $>38^{\circ} \mathrm{C}$ and $<36^{\circ} \mathrm{C}$ ). In order to accurately predict the future development of such an inflammatory state, an accurate prediction of the patient's future temperature is therefore required. Gaussian Processes are a machine learning technique that has been used to model and forecast real dynamic systems, because of their flexible modelling abilities and their high predictive performances. They also allow for multi-dimensional inputs, and they assign a confidence value to their prediction ${ }^{3}$. These properties make Gaussian Processes an interesting forecasting tool in the intensive care domain.

Purpose: Compute accurate predictions of future values of a patient's temperature in blood by means of Gaussian Processes.

Material and Methods: Data consists of physiological variables measured every minute in a PDMS system (Metavision, iMD-Soft), for a set of 30 ICU patients, with lengths of stay varying from a couple of hours to several days. These time series are compressed to obtain a measurement every hour. The following variables are selected as inputs for the Gaussian Process algorithm because of their clinical relevance in prediction of temperature: Blood Temperature, Peripheral temperature, Oxygen blood saturation, Heart Frequency, Mean blood pressure, Systolic blood pressure, PH, Cardiac output, Urine output, Frequency of respiration (measured automatically by the respirator), White blood cell count and Arterial CO2 tension. A fraction of the data from each patient is used to train the Gaussian Process model and the remaining data is used for testing the model's predictive performance. A window of 15 previous time points per physiological variable is used to predict the patient's blood temperature of the following hour. This one-step ahead process is iterated to obtain predictions multiple time steps ahead. To deal with the large amount of data a sparse method (Pseudo-input Gaussian Process) is used.

Results: Predictions 4 and 10 time steps ahead were bounded to an mse below 0.21 and 0.83 respectively for all patients. Predicted standard deviations remained below 0.63 . Using the current value as a predictor of the future temperature resulted in upper bounds for mse of 0.63 and 3.24 for 4 and 10 time steps ahead respectively. Using the average temperature as a predictor the upper bound was 2.77. For all patients, Gaussian Processes outperformed the other

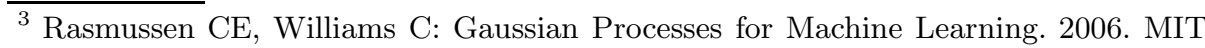
Press: Cambridge
} 
two predictors within the 10 hour ahead prediction range. Predictions for one example patient are shown in Fig1, with performance (mse) shown in Table1.

Conclusion: Gaussian Process when used for forecasting an ICU patient's blood temperature, result in predictions with small mse (within the studied range), when compared to using the mean value or the current value as predictors. The range of prediction must be further extended before these models can be used in prediction of inflammatory states several days in advance.

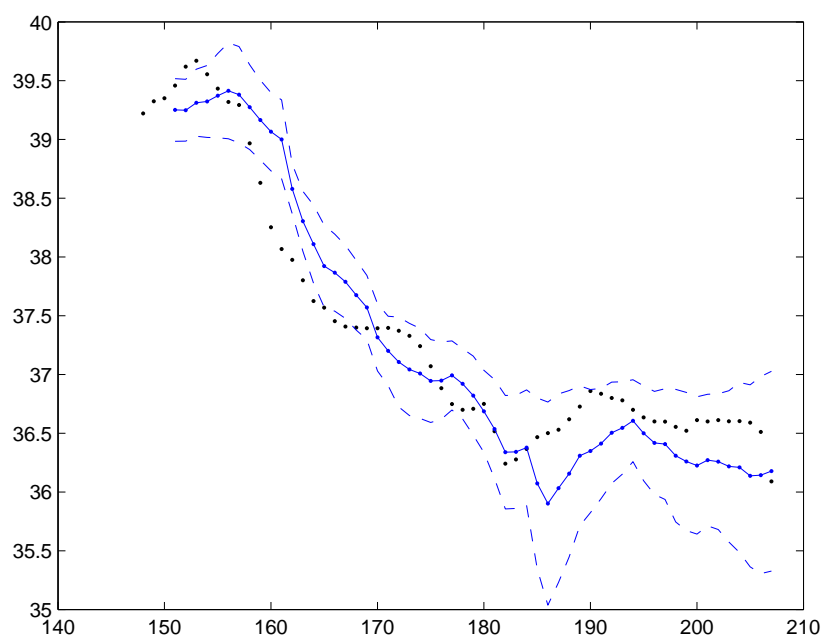

Fig. 1. Four hours ahead prediction of Blood Temperature. Dots are real values and connected dots are predicted values. Dashed lines denote predicted $95 \%$ confidence intervals

\begin{tabular}{|c|c|c|c|}
\hline Steps Ahead & Gaussian Process & Current Value & Mean Value \\
\hline 4 & 0.19 & 0.27 & 2.08 \\
10 & 0.78 & 1.18 & 2.02 \\
24 & 2.81 & 4.07 & 2.00 \\
\hline
\end{tabular}

Table 1. Mean squared errors (mse) of predictions for example patient 